CIRJE-F-519

\title{
Detail-Free Mechanism Design in Twice Iterative Dominance: Large Economies
}

\author{
Hitoshi Matsushima \\ University of Tokyo
}

September 2007

CIRJE Discussion Papers can be downloaded without charge from: http://www.e.u-tokyo.ac.jp/cirje/research/03research02dp.html

Discussion Papers are a series of manuscripts in their draft form. They are not intended for circulation or distribution except as indicated by the author. For that reason Discussion Papers may not be reproduced or distributed without the written consent of the author. 


\title{
Detail-Free Mechanism Design
}

\section{in Twice Iterative Dominance: Large Economies ${ }^{+}$}

\author{
Hitoshi Matsushima* \\ Faculty of Economics, University of Tokyo
}

May 20, 2007

(First Version: June 14, 2004)

\footnotetext{
${ }^{+}$The title of the first version was "Large Auction Design in Dominance" (CIRJE-F-282, University of Tokyo: http://www.e.u-tokyo.ac.jp/cirje/research/dp/2004/2004cf282.pdf). This research was supported by a Grant-in-Aid for Scientific Research (KAKENHI 15330036, 18330035) from the Japan society for the promotion of science (JSPS) and MEXT of the Japanese Government and a grant from the Center for Advanced Research in Finance (CARF) at the University of Tokyo. I am grateful to the anonymous referees for their helpful comments. All errors are mine.

* Faculty of Economics, University of Tokyo, Hongo, Bunkyo-Ku, Tokyo 113, Japan. Fax: +81-3-5841-5521. E-mail: hitoshi@e.u-tokyo.ac.jp
} 


\begin{abstract}
This paper investigates unique implementation in large economies with incomplete information and interdependent values; we degenerate the common knowledge assumptions and assume that a central planner is unaware of the specifications of an environment. With a minor restriction on the class of environments, we demonstrate that there exists a detail-free mechanism that virtually implements competitive allocations with complete information in twice iterative dominance, irrespective of how the environment is specified.
\end{abstract}

Keywords: Large Economies, Interdependent Values, Unique Implementation, Detail-Free Mechanisms, Twice Iterative Dominance.

Journal of Economic Literature Classification Numbers: C70, D60, D78, D82

\title{
1. Introduction
}

This paper investigates the unique implementation problem in economic environments with incomplete information and interdependent values, wherein a sufficiently large number of players exist with single-unit supplies and demands. Each player receives her respective private signals that are correlated with each other through some unobservable macro shock. Each player's value for the commodity depends not only on her private signal but also on this macro shock, which implies interdependent values. The central planner 
attempts to design a well-behaved mechanism in that, irrespective of the macro shock that occurs, every strategy profile that satisfies a defined equilibrium concept can induce a competitive allocation with complete information at least approximately and almost certainly.

The standard approach in the implementation literature with incomplete information has assumed that the central planner has complete knowledge of the specifications of the environments, such as the payoff and signal structures, and tailors a mechanism for the finer details of these specifications. See Jackson (1991), Palfrey (1992), Matsushima (1990, 1993), Abreu and Matsushima (1992, 1994), and Maskin and Sjöström (2002). Equilibrium concepts such as the Nash equilibrium and iterative dominance have generally been used; these concepts rely on the strong rationality assumption that it is not only mutual knowledge but also common knowledge for the players to avoid playing dominated strategies. These assumptions are regarded as the drawbacks of the standard implementation problem and have been criticized from the practical and experimental viewpoints. In fact, many authors studying auction design from the practical standpoint have confined their attention to auction protocols that are detail-free, i.e., independent of the finer details of the specifications. See Krishna (2002), Milgrom (2004), and Klemperer (2004). Several experimental studies have reported that subjects in laboratory experiments like guessing games made a maximum of two or three iterative removals of dominated strategies. For instance, see Camerer (2003, Chapter 5).

Based on the above viewpoints, this paper considers the situation in which the central planner is unaware of the specifications of the environment. From the practical viewpoint, 
we consider the central planner who, instead of tailoring a mechanism based on the specifications, attempts to design a detail-free mechanism that implements the desired allocations irrespective of how the environment is specified. ${ }^{1}$ Further, based on the experimental viewpoint, we use the equilibrium concept of twice iterative dominance, which requires players to eliminate dominated strategies only two times iteratively. Twice iterative dominance is based solely on the weak rationality assumption that it is not common but mutual knowledge among the players that they do not play dominated strategies. The main theorem of this paper is permissive, which reveals that with a minor restriction on the class of possible environments, there exists a detail-free mechanism that can virtually implement competitive allocations with complete information in twice iterative dominance, irrespective of how the environment is specified.

The constructed mechanism describes the following three-stage procedure. The central planner divides the players into a sufficient number of distinct groups, each of which includes an adequate number of sellers and buyers. The central planner then requires each player to announce three price bids as follows. In stage 1 , each player announces the first bid. After the first bid announcement, she observes the first bid announcements in the preceding group. In stage 2, she announces the second bid. After the second bid announcement, she observes the first bid announcements in all groups other than her own.

\footnotetext{
${ }^{1}$ The detail-free concept in this paper implies that the mechanisms are independent of the fine details of value functions, signal spaces, and probability functions. The mechanisms, however, depend on several model settings, such as the finiteness of the signal spaces, single unit demands and supplies, and the numbers of sellers and buyers.
} 
Finally, in stage 3, she announces the third bid.

In order to determine the allocation, the central planner randomly uses two rules: the random fixed price rule and the price-taking rule. With a positive but very low probability, the central planner uses the random fixed price rule, according to which she selects a trading price randomly and independent of the players' announcements. The random fixed price rule incentivizes the players to announce their expected values honestly. In particular, their first bids completely reveal their private signals. Further, on account of the law of large numbers, their second and third bids can approximate their true values associated with the private signals and the unobservable macro shock.

With a very high probability, the central planner uses the price-taking rule instead of the random fixed price rule. The central planner balances the transfers within each group. For each group, she calculates the market-clearing price that equalizes the supplies and demands associated with the second bids within that group. In order to conduct the transfers within each group, the central planner sets the trading price for this group such that it is equal to the market-clearing price for its preceding group and accords the priority to players whose third bids are greater than this price. Since the trading price for each group is determined independent of the announcements in this group, and the signals possessed by the other players in this group are relatively uninformative with respect to all the signals possessed by the players in the other groups, it follows that all players in this group are willing to adopt price-taking behaviors in the virtual sense for their third bid announcements. Hence, they have the incentive to almost honestly announce their conditional expected values that approximate their true values. 
The standard analysis of competitive markets with interdependent values has assumed the form of a non-strategic price taker and investigated a rational expectation equilibrium; for instance, see Mas-Colell, Whinston, and Green (1995, Chapter 19). Each trader updates her belief based on the market price and the forecast function that maps private signal profiles to trading prices, and then maximizes the updated expected utility as a non-strategic price taker. In order to demonstrate a strategic foundation for a rational expectation equilibrium, Reny and Perry (2006) investigated the standard model of a large double auction and established the existence of a Bayesian Nash equilibrium that resembles the rational expectation equilibrium. Also, see McLean, Peck, and Postlewaite (2005). These studies have not considered the issue of uniqueness, which is the central theme of the implementation theory. Moreover, since the trading price for each player is determined independent of her price bids, we do not require any price grid device to restrict the players’ price manipulations as employed by Reny and Perry (2006).

In the implementation literature, Matsushima (2006a) investigated the possibility that a detail-free mechanism implements social choice functions in general environments. Matsushima (2006a) assumed that each player has an intrinsic preference for honest reporting. This paper focuses on players who are motivated only by their material interests.

Another paper by Matsushima (2006b) investigated implementation possibilities of efficient allocations on the assumption that players can conduct a maximum of three iterative removals of dominated strategies. Matsushima (2006b) used mechanisms that are not detail-free and therefore depend on the finer details of the specifications.

Bergemann and Morris (2005a, 2005b) studied the robustness of mechanism design on 
the assumption that the environment is not common knowledge among the players and the central planner. In contrast, the present paper permits the common knowledge assumption among the players with respect to the environment and focuses on the case in which the central planner is unaware of the environment specifications.

The remainder of this paper is organized as follows. Section 2 presents definitions and notations. Section 3 demonstrates the main theorem. Section 4 presents a brief sketch of the proof of the main theorem. Section 5 presents the full proof.

\section{The Model}

Let $n$ and $r$ denote two positive integers. Let $N=\{1, \ldots, n r\} \bigcup\{n r+1, \ldots, 2 n r\}$ denote the set of players, where the first $n r$ players are regarded as the sellers and the last $n r$ players are regarded as the buyers. We assume that both $n$ and $r$ are sufficiently large. Each seller possesses one unit of the commodity, and therefore, the total amount of the commodity equals $n r$. Let $A \subset\{0,1\}^{2 n r}$ denote the set of alternatives, where $a \equiv\left(a_{i}\right)_{i \in N} \in\{0,1\}^{2 n r}$ is included in $A$ if and only if $\sum_{i \in N} a_{i}=n r$, and $a_{i}=1 \quad\left(a_{i}=0\right)$ implies that player $i$ consumes one (zero) unit of the commodity. Let $\Delta(A)$ denote the set of lotteries over alternatives. A mechanism is defined by $G=(n, r, M, g, x)$, where $M=\prod_{i \in N} M_{i}, \quad M_{i}$ is the set of messages for player $i \in N, \quad m_{i} \in M_{i}, \quad m=\left(m_{i}\right)_{i \in N} \in M$, $g: M \rightarrow \Delta(A), \quad x=\left(x_{i}\right)_{i \in N}, \quad x_{i}: M \rightarrow R$, and $x_{i}(m)$ implies the monetary transfer to 
player $i$. We focus on mechanisms that are budget-balancing in $\sum_{i \in N} x_{i}(m)=0$ for all $m \in M$. The central planner chooses any alternative $a \in A$ with probability $g(m)(a) \in[0,1]$.

Each player observes a private signal, $\omega_{i}$. Let $\Omega_{i}$ denote the finite set of player $i$ 's private signals, where we assume that $\Omega_{i}=\Omega_{1}$ for all $i \in N$. Let $\omega=\left(\omega_{i}\right)_{i \in N}$ denote a private signal profile, and let $\Omega=\underset{i \in N}{\times} \Omega_{i}$ denote a set of private signal profiles. Let $\omega_{-i}=\left(\omega_{j}\right)_{j \in N \backslash\{i\}}$ and $\Omega_{-i}=\underset{j \in N \backslash\{i\}}{\times} \Omega_{j}$. We assume that there exists a macro shock $\omega_{0}$ that is unobservable to the players. Let $\Omega_{0}$ denote the finite set of macro shocks. A combination of the private signal profile and the macro shock $\left(\omega_{0}, \omega\right)$ is randomly determined according to the probability function $f: \Omega_{0} \times \Omega \rightarrow(0,1]$. We assume conditional independence in that there exist $f_{0}: \Omega_{0} \rightarrow(0,1]$ and $f_{1}\left(\cdot \mid \omega_{0}\right): \Omega_{1} \rightarrow(0,1]$ for each $\omega_{0} \in \Omega_{0}$ such that

$$
f\left(\omega_{0}, \omega\right)=f_{0}\left(\omega_{0}\right) \prod_{i \in N} f_{1}\left(\omega_{i} \mid \omega_{0}\right) .^{2}
$$

\footnotetext{
${ }^{2}$ Reny and Perry (2006) assumed the conditional independence in the same manner as in this paper, where each player's private signal is determined according to the identical conditional distribution function. However, we can relax this assumption by permitting the sellers' private signals and the buyers’ private signals to be determined according to the different conditional distribution functions. The paper by Fudenberg, Mobius, and Szeidl (2007) investigated large double auctions on this
} 
The value function is defined by $v: \Omega_{0} \times \Omega_{1} \rightarrow(0,1] .{ }^{3}$ Each player $i$ 's value for one unit of the commodity depends on the macro shock $\omega_{0} \in \Omega_{0}$ as well as her private signal $\omega_{i} \in \Omega_{i}$, and is given by $v\left(\omega_{0}, \omega_{i}\right) \in(0,1]$. We assume quasi-linearity and risk neutrality in that the player i's utility is given by $a_{i} v\left(\omega_{0}, \omega_{i}\right)+t_{i}$, where $t_{i} \in R$ is the monetary transfer to player $i$.

A combination $e \equiv\left(\Omega_{0}, \Omega_{1}, f_{0}, f_{1}, v\right)$ defines an environment. We use $E$ to denote a set of environments that satisfies the following three assumptions, which are generically very weak restrictions.

Assumption 1: For every $\omega_{0} \in \Omega_{0}$ and every $\omega_{0}^{\prime} \in \Omega_{0} \backslash\left\{\omega_{0}\right\}$,

$$
f_{1}\left(\cdot \mid \omega_{0}\right) \neq f_{1}\left(\cdot \mid \omega_{0}^{\prime}\right) .
$$

Assumption 1 implies that the different macro shocks correspond to the different probability distributions of the private signal.

Assumption 2: For every $\omega_{0} \in \Omega_{0}$ and every $v \in(0,1]$,

$$
\sum_{\omega_{1} \in \Omega_{1}: v\left(\omega_{0}, \omega_{1}\right) \geq v} f_{1}\left(\omega_{1} \mid \omega_{0}\right) \neq \frac{1}{2}
$$

relaxed conditional independence assumption; however, this paper focused only on the private value cases.

3 The mapping into the interval $[0,1]$ simply implies that the payoffs are uniformly bounded. 
Since $\Omega_{0}$ and $\Omega_{1}$ are finite sets, it holds generically in the class of conditional distribution functions $f_{1}$ that for every $\omega_{0} \in \Omega_{0}$, there exists no subset $\Gamma \subset \Omega_{1}$ such that $\sum_{\omega_{1} \in \Gamma} f_{1}\left(\omega_{1} \mid \omega_{0}\right)=\frac{1}{2}$, which automatically implies Assumption 2.

Let

$$
v\left(\omega_{i}\right) \equiv \sum_{\omega_{0} \in \Omega_{0}} v\left(\omega_{0}, \omega_{i}\right) \tilde{f}\left(\omega_{0} \mid \omega_{i}\right)
$$

denote the expected value conditional on the private signal $\omega_{i} \in \Omega_{i}$, where

$$
\tilde{f}\left(\omega_{0} \mid \omega_{i}\right) \equiv \frac{f_{1}\left(\omega_{i} \mid \omega_{0}\right) f_{0}\left(\omega_{0}\right)}{\sum_{\omega_{0}^{\prime} \in \Omega_{0}} f_{1}\left(\omega_{i} \mid \omega_{0}^{\prime}\right) f_{0}\left(\omega_{0}^{\prime}\right)}
$$

is the probability of the macro shock $\omega_{0}$ occurring conditional on the private signal $\omega_{i} \in \Omega_{i}$.

Assumption 3: For every $\omega_{1} \in \Omega_{1}$ and every $\omega_{1}^{\prime} \in \Omega_{1} \backslash\left\{\omega_{1}\right\}$,

$$
v\left(\omega_{1}\right) \neq v\left(\omega_{1}^{\prime}\right) .
$$

Since $\Omega_{1}$ is a finite set, it holds generically in the class of environments that the different private signals correspond to the different expected values, which is exactly the same as what Assumption 3 implies.

A strategy for player $i \in N$ is denoted by $s_{i}: \Omega_{i} \rightarrow M_{i}$. Let $S_{i}$ denote the set of 
strategies for player $i$. Let $S \equiv \underset{i \in N}{\times} S_{i}, \quad s=\left(s_{i}\right)_{i \in N} \in S$, and $s(\omega)=\left(s_{i}\left(\omega_{i}\right)\right)_{i \in N} \in M$. The expected utility for player $i$ when the players play a strategy profile $s \in S$ is denoted by

$$
u_{i}(s) \equiv \sum_{\left(\omega_{0}, \omega\right) \in \Omega_{0} \times \Omega}\left\{\sum_{a \in A} a_{i} v\left(\omega_{0}, \omega_{i}\right) g(s(\omega))(a)+x_{i}(s(\omega))\right\} f\left(\omega_{0}, \omega\right) .
$$

A strategy $s_{i} \in S_{i}$ for player $i$ is said to be undominated in $(G, e)$ if there exists no $s_{i}^{\prime} \in S_{i}$ such that

$$
u_{i}\left(s_{i}^{\prime}, s_{-i}\right)>u_{i}(s) \text { for all } s_{-i} \in S_{-i} \text {. }
$$

Let $S_{i}^{1} \subset S_{i}$ denote the set of undominated strategies for player $i$ in $(G, e)$. A strategy $s_{i} \in S_{i}$ for player $i$ is said to be twice iteratively undominated in $(G, e)$ if $s_{i} \in S_{i}^{1}$ and there exists no $s_{i}^{\prime} \in S_{i}^{1}$ such that

$$
u_{i}\left(s_{i}^{\prime}, s_{-i}\right)>u_{i}(s) \text { for all } s_{-i} \in S_{-i}^{1} \text {. }
$$

For every environment $e \in E$ and every macro shock $\omega_{0} \in \Omega_{0}$, we define $p^{*}\left(\omega_{0}, e\right) \in[0,1]$ by

$$
\sum_{\omega_{1} \in \Omega_{1}: v\left(\omega_{0}, \omega_{1}\right)<p^{*}\left(\omega_{0}, e\right)} f_{1}\left(\omega_{1} \mid \omega_{0}\right)<\frac{1}{2} \text { and } \sum_{\omega_{1} \in \Omega_{1}: v\left(\omega_{0}, \omega_{1}\right)>p^{*}\left(\omega_{0}, e\right)} f_{1}\left(\omega_{1} \mid \omega_{0}\right)<\frac{1}{2},
$$

where we used Assumption 2 to guarantee its existence. ${ }^{4}$ Since the total amount of the

${ }^{4}$ Note from Assumption 2 that for each $\omega_{0} \in \Omega_{0}$, there exists $\omega_{1} \in \Omega_{1}$ such that $v\left(\omega_{0}, \omega_{1}\right)=p^{*}\left(\omega_{0}, e\right)$. This ensures that with sufficient players, it is almost certain that the trading price is set equal to $p^{*}\left(\omega_{0}, e\right)$ in the mechanisms constructed in this paper. With the exception of this point, Assumption 2 is not considerably relevant to the main theorem of this paper. 
commodity $n r$ is merely half the number of the players $2 n r$, we can regard $p^{*}\left(\omega_{0}, e\right)$ as the competitive price at the macro shock $\omega_{0} \in \Omega_{0}$ in the environment $e \in E$ when the number of players is sufficiently large. Note that the proportion of the players who observe the private signal $\omega_{1}$ is approximated by $f_{1}\left(\omega_{1} \mid \omega_{0}\right)$. Hence, the first inequality implies that less than a half of the players are willing to sell the commodity for the price $p^{*}\left(\omega_{0}, e\right)$. The second inequality implies that less than a half of the players are willing to buy the commodity for $p^{*}\left(\omega_{0}, e\right)$. The rest of the players are indifferent to buying the commodity for $p^{*}\left(\omega_{0}, e\right)$. This implies that $p^{*}\left(\omega_{0}, e\right)$ equalizes the demand and supply.

\section{The Theorem}

Fix an infinite sequence $\left(n^{(l)}, r^{(l)}\right)_{l=1}^{\infty}$ arbitrarily, where $n^{(l)}$ and $r^{(l)}$ are increasing with respect to $l$, and $\lim _{l \rightarrow \infty}\left(n^{(l)}, r^{(l)}\right)=(\infty, \infty)$. An infinite sequence of mechanisms is denoted by $\left(G^{(l)}\right)_{l=1}^{\infty}$, where $G^{(l)}=(n, r, M, g, x)$ and $(n, r)=\left(n^{(l)}, r^{(l)}\right)$ for all $l \geq 1$.

The Theorem: There exists an infinite sequence of mechanisms $\left(G^{(l)}\right)_{l=1}^{\infty}$ such that for every $e \in E$, every $\varepsilon>0$, and every sufficiently large $l \geq 1$, any twice iteratively undominated strategy profile $s \in S$ in $\left(G^{(l)}, e\right)$ satisfies the following properties. Fix $i \in N$ and $\left(\omega_{0}, \omega_{i}\right) \in \Omega_{0} \times \Omega_{i}$ arbitrarily. 
(i) Suppose $v\left(\omega_{0}, \omega_{i}\right)>p^{*}\left(\omega_{0}, e\right)$; then,

$$
\sum_{\omega_{-i} \in \Omega_{-i}} \sum_{a \in A} a_{i} g(s(\omega))(a) f_{-i}\left(\omega_{-i} \mid \omega_{0}\right) \geq 1-\varepsilon,
$$

$$
\sum_{\omega_{-i} \in \Omega_{-i}:: x_{i}(s(\omega)) \mid \leq \varepsilon} f_{-i}\left(\omega_{-i} \mid \omega_{0}\right) \geq 1-\varepsilon \quad \text { if } i \in\{1, \ldots, n r\},
$$

and

$$
\sum_{\omega_{-i} \in \Omega_{-i}:\left|x_{i}(s(\omega))+p^{*}\left(\omega_{0}, e\right)\right| \leq \varepsilon} f_{-i}\left(\omega_{-i} \mid \omega_{0}\right) \geq 1-\varepsilon \quad \text { if } i \in\{n r+1, \ldots, 2 n r\} .
$$

(ii) Suppose $v\left(\omega_{0}, \omega_{i}\right)<p^{*}\left(\omega_{0}, e\right)$; then,

$$
\sum_{\omega_{-i} \in \Omega_{-i}} \sum_{a \in A} a_{i} g(s(\omega))(a) f_{-i}\left(\omega_{-i} \mid \omega_{0}\right) \leq \varepsilon,
$$

$$
\sum_{\omega_{-i} \in \Omega_{-i}:: x_{i}(s(\omega))-p^{*}\left(\omega_{0}, e\right) \leq \varepsilon} f_{-i}\left(\omega_{-i} \mid \omega_{0}\right) \geq 1-\varepsilon \quad \text { if } i \in\{1, \ldots, n r\},
$$

and

$$
\sum_{\omega_{-i} \in \Omega_{-i}:\left|x_{i}(s(\omega))\right| \leq \varepsilon} f_{-i}\left(\omega_{-i} \mid \omega_{0}\right) \geq 1-\varepsilon \quad \text { if } i \in\{n r+1, \ldots, 2 n r\} \text {, }
$$

where $f_{-i}\left(\omega_{-i} \mid \omega_{0}\right) \equiv \prod_{j \in N \backslash\{i\}} f_{1}\left(\omega_{j} \mid \omega_{0}\right)$ denotes the probability of $\omega_{-i} \in \Omega_{-i}$ occurring conditional on $\omega_{0} \in \Omega_{0}$.

The left-hand side of inequalities (1) and (4) represents the probability of player $i$ consuming one unit of the commodity at the macro shock $\omega_{0}$. Inequality (1) implies that any player $i \in N$ almost certainly consumes the commodity if $v\left(\omega_{0}, \omega_{i}\right)>p^{*}\left(\omega_{0}, e\right)$. Inequality (4) implies that any player $i \in N$ rarely consumes the commodity if 
$v\left(\omega_{0}, \omega_{i}\right)<p^{*}\left(\omega_{0}, e\right)$.

The left-hand side of inequalities (2) and (6) represents the probability that the monetary transfer to player $i$ is close to zero. Inequality (2) implies that it is almost certain that the monetary transfer to any seller $i \in\{1, \ldots, n r\}$ is close to zero if $v\left(\omega_{0}, \omega_{i}\right)>p^{*}\left(\omega_{0}, e\right)$. Inequality (6) implies that it is almost certain that the monetary transfer to any buyer $i \in\{n r+1, \ldots, 2 n r\}$ is close to zero if $v\left(\omega_{0}, \omega_{i}\right)<p^{*}\left(\omega_{0}, e\right)$.

The left-hand side of inequality (3) represents the probability that the monetary transfer to player $i$ is close to $-p^{*}\left(\omega_{0}, e\right)$. Inequality (3) implies that it is almost certain that the monetary transfer to any buyer $i \in\{n r+1, \ldots, 2 n r\}$ is close to $-p^{*}\left(\omega_{0}, e\right)$ if $v\left(\omega_{0}, \omega_{i}\right)>p^{*}\left(\omega_{0}, e\right)$. The left-hand side of inequality (5) represents the probability that the monetary transfer to player $i$ is close to $p^{*}\left(\omega_{0}, e\right)$. Inequality (5) implies that it is almost certain that the monetary transfer to any seller $i \in\{1, \ldots, n r\}$ is close to $p^{*}\left(\omega_{0}, e\right)$ if $v\left(\omega_{0}, \omega_{i}\right)<p^{*}\left(\omega_{0}, e\right)$

Based on the above observations, the theorem states that when the number of players is sufficiently large, competitive allocations with complete information are virtually implementable in twice iterative dominance. Moreover, the theorem requires the used mechanisms to be detail-free, i.e., to be designed independent of the specifications of the environments.

The proof of this theorem is constructive; an outline of the proof is provided in the following section. 


\section{Outline of the Proof}

The central planner divides the players into $r$ distinct groups. Each group $\beta \in\{1, \ldots, r\}$ is defined as

$$
N(\beta) \equiv\{(\beta-1) n+1, \ldots, \beta n\} \bigcup\{(r+\beta-1) n+1, \ldots,(r+\beta) n\} \subset N,
$$

which includes $n$ sellers and $n$ buyers. ${ }^{5}$ Let $\beta(i) \in\{1, \ldots, r\}$ denote the index of the group that includes player $i$, i.e., $i \in \beta(i)$. We consider the following three-stage procedure, according to which the central planner requires each player to announce price bids thrice.

Stage 1: Each player $i$ announces the first bid $m_{i, 1} \in[0,1]$ and observes the first bid announcements $m_{N(\beta(i)-1), 1} \equiv\left(m_{j, 1}\right)_{j \in N(\beta(i)-1)} \in[0,1]^{n}$ in the preceding group $\beta(i)-1$.

Stage 2: Each player $i$ announces the second bid $m_{i, 2}\left(m_{N(\beta(i)-1), 1}\right) \in[0,1]$. After this

\footnotetext{
${ }^{5}$ This paper assumes that the numbers of buyers and sellers are the same. The theorem, however, does not depend on this assumption. Suppose that the number of buyers is $n_{b} r$ and that of sellers is $n_{s} r$ and $n_{b} \neq n_{s}$. In this case, we can show the results of the theorem without any substantial change by defining $r$ distinct groups, each of which consists of $n_{b}$ buyers and $n_{s}$ sellers and replicates the whole market.
} 
announcement, each player $i$ observes the first bid announcements $m_{N \backslash N(\beta(i)), 1} \equiv\left(m_{j, 1}\right)_{j \in N \backslash N(\beta(i))} \in[0,1]^{n(r-1)}$ in all groups other than group $\beta(i)$.

Stage 3: Each player $i$ announces the third bid $m_{i, 3}\left(m_{N \backslash N(\beta(i)), 1}\right) \in[0,1]$.

During this procedure, each player $i \in N$ cannot observe the first bid announcements of the other players in the same group $\beta(i)$ and the second bid announcements of all the other players. The central planner determines an allocation and monetary transfers according to the random fixed price rule and the price-taking rule as follows. The central planner generally employs the price-taking rule. The random fixed price rule is used only with a positive but very low probability. As shown later in this paper, the first bid announcements reveal the private signals truthfully; the second bid announcements are utilized to calculate the competitive price with complete information; and the third bid announcements are generally the basis for determining the allocation.

Random Fixed Price Rule: The central planner randomly selects a trading price $p \in[0,1]$. Consider the following six cases:

$$
\begin{aligned}
& m_{i, 1}<p, \quad m_{n r+i, 1}>p, \\
& m_{i, 2}\left(m_{N(\beta(i)-1), 1}\right)<p, \quad m_{n r+i, 2}\left(m_{N(\beta(i)-1), 1}\right)>p, \\
& m_{i, 3}\left(m_{N \backslash N(\beta(i)), 1}\right)<p, \text { and } m_{n r+i, 3}\left(m_{N \backslash N(\beta(i)), 1}\right)>p .
\end{aligned}
$$

For each $k \in\{0, \ldots, 6\}$, each seller $i$ sells the commodity to buyer $i+n r$ for $p$ with a 
probability of $k / 6$ if and only if any $k$ cases among these six cases occur at once.

Price-Taking Rule: The central planner calculates the market-clearing price $p(\beta) \in[0,1]$ for each group $\beta \in\{1, \ldots, r\}$, which is defined as the $n$th highest bid among the second bids announced in group $\beta$. Within each group $\beta$, the central planner sets the trading price for the group equal to the market-clearing price $p(\beta-1)$ of its preceding group $\beta-1$. Every seller in each group $\beta$ is compelled to sell the commodity to the central planner for $p(\beta-1)$. The central planner then sells these $n$ units back to the players in the same group $\beta$ whose third bids are greater than $p(\beta-1)$. If less than $n$ players in group $\beta$ make their third bids greater than $p(\beta-1)$, the central planner randomly selects and compels players to buy the unsold commodities. If more than $n$ players make their third bids greater than $p(\beta-1)$, the central planner randomly selects and sells the commodities to $n$ players among them.

Note that the above procedure is detail-free, i.e., it does not depend on the finer details of the specifications. Fix an environment $e \in E$ arbitrarily. We show that each player's first bid reveals her private signal truthfully. Note that each player's first bid influences her payoff only through the random fixed price rule. Since the trading price $p$ is randomly determined, each player $i$ is willing to make the first bid $m_{i, 1}$ equal to the expected value $v_{i}\left(\omega_{i}\right)$ conditional on her private signal $\omega_{i}$ as being undominated. This, along with 
Assumption 3, implies that each player's first bid reveals her private signal truthfully.

Next, we show that, almost certainly, each player i's second bid approximates the true value $v\left(\omega_{i}, \omega_{0}\right)$, and the market-clearing price $p(\beta)$ for each group $\beta$ approximates the competitive price $p^{*}\left(\omega_{0}, e\right)$. Note that each player's second bid influences her payoff only through the random fixed price rule. Before announcing the second bid, each player $i$ has complete knowledge of the private signals $\omega_{N(\beta(i)-1)} \equiv\left(\omega_{j}\right)_{j \in N(\beta(i)-1)} \in \Omega_{1}^{n}$ for the players in the preceding group $\beta(i)-1$. Since the trading price $p$ is randomly determined, it follows that each player $i$ is willing to make the second bid $m_{i, 2}\left(m_{N(\beta(i)-1), 1}\right)$ equal to the expected value conditional on the private signals $\omega_{N(\beta(i)-1)}$ for the members of the preceding group $\beta(i)-1$ and her private signal $\omega_{i}$, i.e.,

$$
v\left(\omega_{i}, \omega_{N(\beta(i)-1)}\right) \equiv \sum_{\omega_{0} \in \Omega_{0}} v\left(\omega_{0}, \omega_{i}\right) \tilde{f}\left(\omega_{0} \mid \omega_{i}, \omega_{N(\beta(i)-1)}\right)
$$

as being twice iteratively undominated, where

$$
\tilde{f}\left(\omega_{0} \mid \omega_{i}, \omega_{N(\beta(i)-1)}\right) \equiv \frac{\prod_{j \in\{i\} \cup N(\beta(i)-1)} f_{1}\left(\omega_{j} \mid \omega_{0}\right) f_{0}\left(\omega_{0}\right)}{\sum_{\omega_{0}^{\prime} \in \Omega_{0}} \prod_{j \in\{i\} \cup N(\beta(i)-1)} f_{1}\left(\omega_{j} \mid \omega_{0}^{\prime}\right) f_{0}\left(\omega_{0}^{\prime}\right)}
$$

denotes the probability of $\omega_{0}$ occurring conditional on $\left(\omega_{i}, \omega_{N(\beta(i)-1)}\right)$. Since the size of each group $2 n$ is sufficiently large, it is almost certain from Assumption 1 that each player can almost correctly infer the true macro shock $\omega_{0}$ from $\omega_{N(\beta(i)-1)}$, and therefore, $v\left(\omega_{i}, \omega_{N(\beta(i)-1)}\right)$ can approximate $v\left(\omega_{i}, \omega_{0}\right)$. Since the size $2 n$ of each group is 
sufficiently large, it is almost certain that each group $\beta$ approximately replicates the whole, which, along with Assumption 2, implies that $p(\beta)$ approximates $p^{*}\left(\omega_{0}, e\right)$.

Finally, we show that each player i's third bid almost certainly approximates the true value $v\left(\omega_{i}, \omega_{0}\right)$, and the competitive allocations are approximately achieved. Before announcing the third bid, each player $i$ has full knowledge of the private signals $\omega_{N \backslash N(\beta(i))} \in \Omega_{1}^{n(r-1)}$ for all players out of group $\beta(i)$. Similarly, the random fixed price rule incentivizes each player $i$ to make the third bid $m_{i, 3}\left(m_{N \backslash N(\beta(i)), 1}\right)$ equal to the expected value conditional on $\omega_{N \backslash N(\beta(i))}$, i.e.,

$$
v\left(\omega_{i}, \omega_{N \backslash N(\beta(i))}\right) \equiv \sum_{\omega_{0} \in \Omega_{0}} v\left(\omega_{0}, \omega_{i}\right) \tilde{f}\left(\omega_{0} \mid \omega_{i}, \omega_{N \backslash N(\beta(i))}\right)
$$

as being twice iteratively undominated, where

$$
\tilde{f}\left(\omega_{0} \mid \omega_{i}, \omega_{N \backslash N(\beta(i))}\right) \equiv \frac{\prod_{j \in\{i\} \cup N \backslash N(\beta(i))} f_{1}\left(\omega_{j} \mid \omega_{0}\right) f_{0}\left(\omega_{0}\right)}{\sum_{\omega_{0}^{\prime} \in \Omega_{0}} \prod_{j \in\{i\} \cup N \backslash N(\beta(i))} f_{1}\left(\omega_{j} \mid \omega_{0}^{\prime}\right) f_{0}\left(\omega_{0}^{\prime}\right)}
$$

denotes the probability of $\omega_{0}$ occurring conditional on $\left(\omega_{i}, \omega_{N \backslash N(\beta(i))}\right)$. Since the size of each group $2 n$ is sufficiently large, it follows from Assumption 1 that $v\left(\omega_{i}, \omega_{N \backslash N(\beta(i))}\right)$ almost certainly approximates $v\left(\omega_{i}, \omega_{0}\right)$.

Since the price-taking rule as well as the random fixed price rule is relevant to the third bid announcement, the above argument is not sufficient for the incentive to reveal any approximate value of $v\left(\omega_{i}, \omega_{0}\right)$ as the third bid announcement. However, we can demonstrate that the price-taking rule does not provide each player with the incentive to 
deviate from revealing any approximate value of the true value $v\left(\omega_{i}, \omega_{0}\right)$ as follows. Let

$$
v\left(\omega_{i}, \omega_{N \backslash\{i\}}\right) \equiv \sum_{\omega_{0} \in \Omega_{0}} v\left(\omega_{0}, \omega_{i}\right) \tilde{f}\left(\omega_{0} \mid \omega\right)
$$

denote the expected value conditional on the private signals for all players, where $\tilde{f}\left(\omega_{0} \mid \omega_{i}, \omega_{N \backslash\{i\}}\right) \equiv \frac{\prod_{j \in N} f_{1}\left(\omega_{j} \mid \omega_{0}\right) f_{0}\left(\omega_{0}\right)}{\sum_{\omega_{0}^{\prime} \in \Omega_{0}} \prod_{j \in N} f_{1}\left(\omega_{j} \mid \omega_{0}^{\prime}\right) f_{0}\left(\omega_{0}^{\prime}\right)}$ is the probability of $\omega_{0}$ occurring conditional on $\left(\omega_{i}, \omega_{N \backslash\{i\}}\right)$. Note that the trading price for each group $\beta$ is set such that it is equal to the market-clearing price $p(\beta-1)$ for its preceding group $\beta-1$, which does not depend on the price bid announcements in group $\beta$. Hence, each player $i$ has the incentive to announce any value between $\min _{\omega_{N(\beta i)) \backslash i i}} v_{i}\left(\omega_{i}, \omega_{N \backslash\{i\}}\right)$ and $\max _{\omega_{N(\beta i) \backslash\{i\}}} v_{i}\left(\omega_{i}, \omega_{N \backslash\{i\}}\right)$ as the third bid. Since the number of distinct groups $r$ is sufficiently large, it follows that both $\min _{\omega_{N(\beta(i)) \backslash i\}}} v_{i}\left(\omega_{i}, \omega_{N \backslash\{i\}}\right)$ and $\max _{\omega_{N(\beta(i)) \backslash i\}}} v_{i}\left(\omega_{i}, \omega_{N \backslash\{i\}}\right)$ are close to $v\left(\omega_{i}, \omega_{N \backslash\{i\}}\right)$. In other words, $v\left(\omega_{i}, \omega_{N \backslash\{i\}}\right)$ does not depend to a large extent on $\omega_{N(\beta(i)) \backslash\{i\}}$, and therefore, $v\left(\omega_{i}, \omega_{N \backslash N(\beta(i))}\right)$ certainly approximates $v\left(\omega_{i}, \omega_{N \backslash\{i\}}\right)$. This, along with the fact that the trading price for each group is independent of the price bid announcements in this group, implies that the price-taking rule does not provide player $i$ with the incentive to deviate from announcing any approximate value of $v\left(\omega_{0}, \omega_{i}\right)$ as her third bid. Note that the third bid is not necessarily equal to $v\left(\omega_{i}, \omega_{N \backslash N(\beta(i))}\right)$; however, it can be approximated by $v\left(\omega_{i}, \omega_{N \backslash N(\beta(i))}\right)$. Since the trading prices almost certainly approximate the competitive price $p^{*}\left(\omega_{0}, e\right)$, we have shown that the competitive allocations with complete information are 
almost certainly implemented in twice iterative dominance. We now present the full proof of the theorem in the following section.

\section{Full Proof of the Theorem}

We model the three-stage procedure in Section 4 as a mechanism $G=G^{(n, r, \varepsilon)}=(n, r, M, g, x)$ as follows. Let $\varepsilon \in(0,1)$,

$$
\begin{aligned}
& M_{i}=M_{i, 1} \times M_{i, 2} \times M_{i, 3}, M_{i, 1}=[0,1], \\
& M_{i, 2} \text { denote the set of continuous functions } m_{i, 2}:[0,1]^{n} \rightarrow[0,1], \\
& M_{i, 3} \text { denote the set of continuous functions } m_{i, 3}:[0,1]^{(r-1) n} \rightarrow[0,1], \\
& g=\frac{\varepsilon}{6}\left(g^{S, 1}+g^{B, 1}+g^{S, 2}+g^{B, 2}+g^{S, 3}+g^{B, 3}\right)+(1-\varepsilon) g^{3}, \text { and } \\
& x_{i}=\frac{\varepsilon}{6}\left(x_{i}^{S, 1}+x_{i}^{B, 1}+x_{i}^{S, 2}+x_{i}^{B, 2}+x_{i}^{S, 3}+x_{i}^{B, 3}\right)+(1-\varepsilon) x_{i}^{3} .
\end{aligned}
$$

Here,

$$
\begin{aligned}
& g^{D, l}: M \rightarrow \Delta(A) \text { for all }(D, l) \in\{S, B\} \times\{1,2,3\}, \text { and } \\
& g^{3}: M \rightarrow \Delta(A) . \\
& x_{i}^{D, l}: M \rightarrow R \text { for all }(D, l) \in\{S, B\} \times\{1,2,3\}, \text { and } \\
& x_{i}^{3}: M \rightarrow R .
\end{aligned}
$$

Each player $i$ simultaneously chooses a message $m_{i}=\left(m_{i, 1}, m_{i, 2}, m_{i, 3}\right)$, where $m_{i, 1} \in[0,1]$ corresponds to the first bid, the value $m_{i, 2}\left(m_{N(\beta(i)-1), 1}\right) \in[0,1]$ of the function $m_{i, 2}$ 
corresponds to the second bid, and the value $m_{i, 3}\left(m_{N \backslash N(\beta(i)), 1}\right) \in[0,1]$ of the function $m_{i, 3}$ corresponds to the third bid. In the following two subsections, we specify $\left(g^{D, l},\left(x_{i}^{D, l}\right)_{i \in N}\right)_{(D, l) \in\{S, B\} \times\{1,2,3\}}$ and $\left(g^{3},\left(x_{i}^{3}\right)_{i \in N}\right)$, which define the random fixed price rule and the price-taking rule, respectively. The central planner uses the random fixed price rule with probability $\varepsilon$ and the price-taking rule with probability $1-\varepsilon$. Hence, the central planner chooses any alternative $a \in A$ with the following probability:

$$
g(m)(a)=\frac{\varepsilon}{6} \sum_{(D, l) \in\{S, B\} \times\{1,2,3\}} g^{D, l}(m)(a)+(1-\varepsilon) g^{3}(m)(a) .
$$

The monetary transfers conducted by the central planner are set such that they are equal to the expected values of the monetary transfers induced by the three-stage procedure. ${ }^{6}$

\subsection{Random Fixed Price Rule}

For every $(m, a) \in M \times A$, let

$$
g^{S, 1}(m)(a)=\prod_{i=1}^{n r} g_{i}^{S, 1}(m)\left(a_{i}, a_{n r+i}\right)
$$

where

\footnotetext{
${ }^{6}$ Here, we assume quasi-linearity and risk neutrality. Without these assumptions, we need to modify the construction of mechanisms by using lotteries over combinations of an alternative and transfers. It might be important to verify the robustness of our results in terms of a non-expected utility, which is beyond the purpose of the present paper.
} 


$$
\begin{aligned}
& g_{i}^{S, 1}(m)(1,0)=m_{i, 1}, \quad g_{i}^{S, 1}(m)(0,1)=1-m_{i, 1}, \\
& g_{i}^{S, 1}(m)(0,0)=0, \text { and } \quad g_{i}^{S, 1}(m)(1,1)=0 .
\end{aligned}
$$

With probability $1-m_{i, 1}$, seller $i$ 's commodity is transferred to buyer $n r+i$. Let

$$
x_{i}^{S, 1}(m) \equiv \int_{m_{i, 1}}^{1} p d p=\frac{1-\left(m_{i, 1}\right)^{2}}{2} \text { and } x_{n r+i}^{S, 1}(m) \equiv-x_{i}^{S, 1}(m)
$$

The central planner randomly selects a price $p$ from the interval $[0,1]$, for which the commodity is transferred from seller $i$ to buyer $n r+i$ if and only if $m_{i, 1}<p$. Note that $x_{i}^{S, 1}(m)$ corresponds to the expected monetary transfer from buyer $n r+i$ to seller $i$. For every $(m, a) \in M \times A$, let

$$
g^{B, 1}(m)(a)=\prod_{i=1}^{n r} g_{i}^{B, 1}(m)\left(a_{i}, a_{n r+i}\right)
$$

where

$$
\begin{aligned}
& g_{i}^{B, 1}(m)(1,0)=1-m_{n r+i, 1}, \quad g_{i}^{B, 1}(m)(0,1)=m_{n r+i, 1}, \\
& g_{i}^{B, 1}(m)(0,0)=0, \text { and } g_{i}^{B, 1}(m)(1,1)=0 .
\end{aligned}
$$

With probability $m_{n r+i, 1}$, seller $i$ 's commodity is transferred to buyer $n r+i$. Let

$$
x_{i}^{B, 1}(m) \equiv \int_{0}^{m_{n r+i, 1}} p d p=\frac{\left(m_{n r+i, 1}\right)^{2}}{2} \text { and } x_{n r+i}^{B, 1}(m) \equiv-x_{i}^{B, 1}(m) .
$$

The central planner randomly selects $p$ from $[0,1]$, for which the commodity is transferred from seller $i$ to buyer $n r+i$ if and only if $m_{n r+i, 1}>p$. Note that $x_{i}^{B, 1}(m)$ corresponds to the expected monetary transfer from buyer $n r+i$ to seller $i$. 
For every $(m, a) \in M \times A$, let

$$
g^{S, 2}(m)(a)=\prod_{i=1}^{n r} g_{i}^{S, 2}(m)\left(a_{i}, a_{n r+i}\right)
$$

where

$$
\begin{aligned}
& g_{i}^{S, 2}(m)(1,0)=m_{i, 2}\left(m_{N(\beta(i)-1), 1}\right), g_{i}^{S, 2}(m)(0,1)=1-m_{i, 2}\left(m_{N(\beta(i)-1), 1}\right), \\
& g_{i}^{S, 2}(m)(0,0)=0, \text { and } g_{i}^{S, 2}(m)(1,1)=0 .
\end{aligned}
$$

With probability $1-m_{i, 2}\left(m_{N(\beta(i)-1), 1}\right)$, seller i's commodity is transferred to buyer $n r+i$.

Let

$$
\begin{aligned}
& x_{i}^{S, 2}(m) \equiv \int_{m_{i, 2}\left(m_{N(\beta(i)-1,1)}\right)}^{1} p d p=\frac{1-m_{i, 2}\left(m_{N(\beta(i)-1), 1}\right)^{2}}{2} \text {, and } \\
& x_{n r+i}^{S, 2}(m) \equiv-x_{i}^{S, 2}(m) .
\end{aligned}
$$

The central planner randomly selects $p$ from $[0,1]$, for which the commodity is transferred from seller $i$ to buyer $n r+i$ if and only if $m_{i, 2}\left(m_{N(\beta(i)-1), 1}\right)<p$. Note that $x_{i}^{S, 2}(m)$ corresponds to the expected monetary transfer from buyer $n r+i$ to seller $i$. For every $(m, a) \in M \times A$, let

$$
g^{B, 2}(m)(a)=\prod_{i=1}^{n r} g_{i}^{B, 2}(m)\left(a_{i}, a_{n r+i}\right)
$$

where

$$
\begin{aligned}
& g_{i}^{B, 2}(m)(1,0)=1-m_{n r+i, 2}\left(m_{N(\beta(i)-1), 1}\right), g_{i}^{B, 2}(m)(0,1)=m_{n r+i, 2}\left(m_{N(\beta(i)-1), 1}\right), \\
& g_{i}^{B, 2}(m)(0,0)=0, \text { and } g_{i}^{B, 2}(m)(1,1)=0 .
\end{aligned}
$$

With probability $m_{n r+i, 2}\left(m_{N(\beta(i)-1), 1}\right)$, seller i's commodity is transferred to buyer $n r+i$. 
Let

$$
\begin{aligned}
& x_{i}^{B, 2}(m) \equiv \int_{0}^{m_{n r+i, 2}\left(m_{N(\beta(i)-1), 1}\right)} p d p=\frac{m_{n r+1,2}\left(m_{N(\beta(i)-1), 1}\right)^{2}}{2} \text {, and } \\
& x_{n r+i}^{B, 2}(m) \equiv-x_{i}^{B, 2}(m) .
\end{aligned}
$$

The central planner randomly selects $p$ from $[0,1]$, for which the commodity is transferred from seller $i$ to buyer $n r+i$ if and only if $m_{n r+i, 2}\left(m_{N(\beta(i)-1), 1}\right)>p$. Note that $x_{i}^{B, 2}(m)$ corresponds to the expected monetary transfer from buyer $n r+i$ to seller $i$.

For every $(m, a) \in M \times A$, let

$$
g^{S, 3}(m)(a)=\prod_{i=1}^{n r} g_{i}^{S, 3}(m)\left(a_{i}, a_{n r+i}\right)
$$

where

$$
\begin{aligned}
& g_{i}^{S, 3}(m)(1,0)=m_{i, 3}\left(m_{N \backslash N(\beta(i)), 1}\right), g_{i}^{S, 3}(m)(0,1)=1-m_{i, 3}\left(m_{N \backslash N(\beta(i)), 1}\right), \\
& g_{i}^{S, 3}(m)(0,0)=0, \text { and } g_{i}^{S, 3}(m)(1,1)=0 .
\end{aligned}
$$

With probability $1-m_{i, 3}\left(m_{N \backslash N(\beta(i)), 1}\right)$, seller $i$ 's commodity is transferred to buyer $n r+i$. Let

$$
\begin{aligned}
& x_{i}^{S, 3}(m) \equiv \int_{m_{i, 3}\left(m_{N \backslash N(\beta(i)), 1}\right)}^{1} p d p=\frac{1-m_{i, 3}\left(m_{N \backslash N(\beta(i)), 1}\right)^{2}}{2} \text {, and } \\
& x_{n r+i}^{S, 3}(m) \equiv-x_{i}^{S, 3}(m) .
\end{aligned}
$$

The central planner randomly selects $p$ from [0,1], for which the commodity is transferred from seller $i$ to buyer $n r+i$ if and only if $m_{i, 3}\left(m_{N \backslash N(\beta(i)), 1}\right)<p$. Note that 
$x_{i}^{S, 3}(m)$ is the expected payment from buyer $n r+i$ to seller $i$. For every $(m, a) \in M \times A$, let

$$
g^{B, 3}(m)(a)=\prod_{i=1}^{n r} g_{i}^{B, 3}(m)\left(a_{i}, a_{n r+i}\right)
$$

where

$$
\begin{aligned}
& g_{i}^{B, 3}(m)(1,0)=1-m_{n r+i, 3}\left(m_{N \backslash N(\beta(i)), 1}\right), g_{i}^{B, 3}(m)(0,1)=m_{n r+i, 3}\left(m_{N \backslash N(\beta(i)), 1}\right), \\
& g_{i}^{B, 3}(m)(0,0)=0, \text { and } g_{i}^{B, 3}(m)(1,1)=0 .
\end{aligned}
$$

With probability $m_{n r+i, 3}\left(m_{N \backslash N(\beta(i)), 1}\right)$, seller $i$ 's commodity is transferred to buyer $n r+i$.

Let

$$
\begin{aligned}
& x_{i}^{B, 3}(m) \equiv \int_{0}^{m_{n r+i, 3}\left(m_{N \backslash N(\beta(i)), 1}\right)} p d p=\frac{m_{n r+1,3}\left(m_{N \backslash N(\beta(i)), 1}\right)^{2}}{2} \text {, and } \\
& x_{n r+i}^{B, 3}(m) \equiv-x_{i}^{B, 3}(m) .
\end{aligned}
$$

The central planner randomly selects $p$ from $[0,1]$, for which the commodity is transferred from seller $i$ to buyer $n r+i$ if and only if $m_{n r+i, 3}\left(m_{N \backslash N(\beta(i)), 1}\right)>p$. Note that $x_{i}^{B, 3}(m)$ corresponds to the expected monetary transfer from buyer $n r+i$ to seller $i$.

\subsection{Price-Taking Rule}

Let $A_{N(\beta)} \equiv \underset{i \in N(\beta)}{\times} A_{i}$ and $a_{N(\beta)}=\left(a_{i}\right)_{i \in N(\beta)} \in A_{N(\beta)}$. For every $(m, a) \in M \times A$, let

$$
g^{3}(m)(a)=\prod_{\beta=1}^{r} g_{\beta}^{3}(m)\left(a_{N(\beta)}\right),
$$


where $g_{\beta}^{3}: M \rightarrow \Delta\left(A_{N(\beta)}\right)$ will be specified later. For every $(\beta, m) \in\{1, \ldots, r\} \times M$, let $\mu(\beta, m):\{1, \ldots, 2 n\} \rightarrow N(\beta)$ denote the one-to-one mapping defined by $m_{\mu(\beta, m)(1), 2}\left(m_{N(\beta-1), 1}\right) \geq m_{\mu(\beta, m)(2), 2}\left(m_{N(\beta-1), 1}\right) \cdots \geq m_{\mu(\beta, m)(2 n), 2}\left(m_{N(\beta-1), 1}\right)$, where for every $l \in\{1, \ldots, 2 n-1\} \quad, \quad$ if $\quad m_{\mu(\beta, m)(l), 2}\left(m_{N(\beta-1), 1}\right)=m_{\mu(\beta, m)(l+1), 2}\left(m_{N(\beta-1), 1}\right) \quad, \quad$ then $\mu(\beta, m)(l)<\mu(\beta, m)(l+1)$. For every $(\beta, m) \in\{1, \ldots, r\} \times M$, let

$$
p(\beta)=p(\beta, m) \equiv m_{\mu(\beta, m)(n), 2}\left(m_{N(\beta-1), 1}\right)
$$

denote the market-clearing price for group $\beta$ that is calculated on the basis of the second bids $\left(m_{i, 2}\left(m_{N(\beta(i)-1), 1}\right)\right)_{i \in N(\beta)}$. Let $n(\beta, p) \in\{1, \ldots, 2 n\}$ denote the integer such that

$$
m_{\mu(\beta, m)(n(\beta, p)), 3}\left(m_{N \backslash N(\beta), 1}\right)>p \geq m_{\mu(\beta, m)(n(\beta, p)+1), 3}\left(m_{N \backslash N(\beta), 1}\right),
$$

which represents the number of players in group $\beta$ whose third bids are greater than $p$. Let $\tilde{n}(\beta, p) \in\{1, \ldots, 2 n\}$ denote the integer such that

$$
m_{\mu(\beta, m)(n(\beta, p)), 3}\left(m_{N \backslash N(\beta), 1}\right) \geq p>m_{\mu(\beta, m)(n(\beta, p)+1), 3}\left(m_{N \backslash N(\beta), 1}\right),
$$

which is the number of players in group $\beta$ whose third bids are greater than or equal to $p$. Clearly, $n(\beta, p) \geq \tilde{n}(\beta, p)$. For each $(\beta, m) \in\{1, \ldots, r\} \times M$, we specify $g_{\beta}^{3}(m)$ as follows. Let $p=p(\beta-1, m)$.

Case 1: Suppose $n(\beta, p) \geq n$. In this case, for every $a_{N(\beta)} \in A_{N(\beta)}$,

$$
\begin{aligned}
g_{\beta}^{3}(m)\left(a_{N(\beta)}\right)= & \frac{n !\{n(\beta, p)-n\} !}{n(\beta, p) !} \\
& \text { if }\left[a_{i}=1\right] \Rightarrow\left[m_{i, 3}\left(m_{N \backslash N(\beta), 1}\right)>p\right] \text { for all } i \in N(\beta),
\end{aligned}
$$


whereas $g_{\beta}^{3}(m)\left(a_{N(\beta)}\right)=0$ otherwise.

Case 2: Suppose $\tilde{n}(\beta, p) \geq n>n(\beta, p)$. Then, for every $a_{N(\beta)} \in A_{N(\beta)}$,

$$
\begin{aligned}
g_{\beta}^{3}(m)\left(a_{N(\beta)}\right)= & \frac{(\tilde{n}(\beta, p)-n) !\{n-n(\beta, p)\} !}{\{\tilde{n}(\beta, p)-n(\beta, p)\} !} \\
& \text { if }\left[a_{i}=1\right] \Rightarrow\left[m_{i, 3}\left(m_{N \backslash N(\beta), 1}\right) \geq p(\beta, m)\right] \text { and } \\
& {\left[m_{i, 3}\left(m_{N \backslash N(\beta), 1}\right)>p\right] \Rightarrow\left[a_{i}=1\right] \text { for all } i \in N(\beta), }
\end{aligned}
$$

whereas $g_{\beta}^{3}(m)\left(a_{N(\beta)}\right)=0$ otherwise.

Case 3: Suppose $n \geq \tilde{n}(\beta, p)$. Then, for every $a_{N(\beta)} \in A_{N(\beta)}$,

$$
\begin{aligned}
g_{\beta}^{3}(m)\left(a_{N(\beta)}\right)= & \frac{n !\{n-\tilde{n}(\beta, p)\} !}{\{2 n-\tilde{n}(\beta, p)\} !} \\
& \text { if }\left[m_{i, 3}\left(m_{N \backslash N(\beta), 1}\right) \geq p\right] \Rightarrow\left[a_{i}=1\right] \text { for all } i \in N(\beta),
\end{aligned}
$$

whereas $g_{\beta}^{3}(m)\left(a_{N(\beta)}\right)=0$ otherwise.

The central planner sets the trading price for each group $\beta$ such that it is equal to the market-clearing price $p(\beta-1)$ for its preceding group $\beta-1$. Those players in each group $\beta$ whose bids are greater than $p(\beta-1)$ have a higher priority to consume the commodity than do the others. Players whose bids are equal to $p(\beta-1, m)$ have a higher priority than those whose bids are less than $p(\beta-1)$. 
One interpretation is as follows. Every seller in each group $\beta$ is compelled to sell the commodity to the central planner for $p(\beta-1)$. The central planner then sells these $n$ units back to the players in the same group $\beta$ whose third bids are greater than $p(\beta-1)$. If the third bids of more than $n$ players are bids greater than $p(\beta-1)$, the central planner randomly selects from among these players and sells the commodities to them. This corresponds to Case 1. If less than $n$ players in group $\beta$ make their third bids greater than or equal to $p(\beta-1)$, the central planner randomly selects from among the rest of the players and compels the selected players to buy the unsold commodities. This corresponds to Case 3. If less than $n$ players make their third bids greater than $p(\beta-1)$ and more than $n$ players in group $\beta$ make their third bids greater than or equal to $p(\beta-1)$, the central planner sells the commodities to the players who make their third bids greater than $p(\beta-1)$, and then randomly selects from among the players who equal their third bids to $p(\beta-1)$ and compels them to buy the unsold commodities. This corresponds to Case 2 .

For every $i \in N(\beta) \cap\{1, \ldots, n r\}$, let

$$
x_{i}^{3}(m)=\left\{1-\sum_{a_{N(\beta)} \in A_{N(\beta)}} a_{i} g_{\beta}^{3}(m)\left(a_{N(\beta)}\right)\right\} p(\beta-1, m) .
$$

For every $i \in N(\beta) \cap\{n r+1, \ldots, 2 n r\}$, let

$$
x_{i}^{3}(m)=-\sum_{a_{N(\beta)} \in A_{N(\beta)}} a_{i} g_{\beta}^{3}(m)\left(a_{N(\beta)}\right) p(\beta-1, m) .
$$

Any buyer $i \in N(\beta)$ who consumes the commodity must pay $p(\beta-1)$. Any seller $i \in N(\beta)$ who does not consume the commodity can receive $p(\beta-1)$. Hence, we have 
completed the construction of the mechanism $G=G^{(n, r, \varepsilon)}=(n, r, M, g, x)$.

Let $\left(\varepsilon^{(l)}\right)_{l=1}^{\infty}$ denote an infinite sequence in which $\varepsilon^{(l)} \in(0,1 / 6), \quad \varepsilon^{(l)}$ decreases with respect to $l$, and $\lim _{l \rightarrow \infty} \varepsilon^{(l)}=0$. For each $l=1,2, \ldots$, let $G^{(l)} \equiv G^{\left(n^{(l)}, r^{(l)}, \varepsilon^{(l)}\right)}$. In the following subsections, we will demonstrate that $\left(G^{(l)}\right)_{l=1}^{\infty}$ satisfies the properties of the theorem.

\subsection{Twice Iterative Dominance}

All the functions relevant to player $i$ 's utility are $g_{i}^{D, l}$ and $x_{i}^{D, l}$ for all $(D, l) \in\{S, B\} \times\{1,2\}, \quad g_{\beta(i)}^{3}$, and $x_{\beta(i)}^{3}$. Suppose that player $i$ is a seller, i.e., $i \in\{1, \ldots, n r\}$. Then, among these functions, only $g_{i}^{S, 1}$ and $x_{i}^{S, 1}$ depend on player $i$ 's first bid $m_{i, 1}$ and only on this bid. According to the specifications of $g_{i}^{S, 1}$ and $x_{i}^{S, 1}$, player $i$ will choose $m_{i, 1}$ to maximize $m_{i, 1} v\left(\omega_{i}\right)+\frac{1-m_{i, 1}^{2}}{2}$, i.e., $m_{i, 1}=v\left(\omega_{i}\right)$. Suppose that player $i$ is a buyer, i.e., $i \in\{n r+1, \ldots, 2 n r\}$. Then, among these functions, only $g_{i}^{B, 1}$ and $x_{i}^{B, 1}$ depend on player $i$ 's first bid $m_{i, 1}$ and only on this bid. According to the specifications of $g_{i}^{B, 1}$ and $x_{i}^{B, 1}$, player $i$ will choose $m_{i, 1}$ to maximize $m_{i, 1} v\left(\omega_{i}\right)-\frac{m_{i, 1}^{2}}{2}$, i.e., $m_{i, 1}=v\left(\omega_{i}\right)$. Hence, we have proved that for every $i \in N$, if $s_{i}$ is undominated, then it must hold that $s_{i, 1}\left(\omega_{i}\right)=v\left(\omega_{i}\right)$ for all $\omega_{i} \in \Omega_{i}$. From Assumption 3, if all players play undominated strategies, then each player's first bid truthfully reveals her private signal. Assuming that all 
players play undominated strategies, their first bids truthfully reveal their private signals.

Suppose that player $i$ is a seller. Then, among the functions $g_{i}^{D, l}$ and $x_{i}^{D, l}$ for all $(D, l) \in\{S, B\} \times\{1,2\}, \quad g_{\beta(i)}^{3}$, and $x_{\beta(i)}^{3}$, only $g_{i}^{S, 2}$ and $x_{i}^{S, 2}$ depend on player $i^{\prime} s$ second bid $m_{i, 2}\left(m_{N(\beta(i)-1), 1}\right)$ and only on this bid. The specifications of $g_{i}^{S, 2}$ and $x_{i}^{S, 2}$, along with the truthful revelation via $m_{N(\beta(i)-1), 1}$, imply that player $i$ will choose $m_{i, 2}\left(m_{N(\beta(i)-1), 1}\right)$ to maximize $\quad m_{i, 2}\left(m_{N(\beta(i)-1), 1}\right) v\left(\omega_{i}, \omega_{N(\beta(i)-1)}\right)+\frac{1-m_{N(\beta(i)-1), 1}^{2}}{2} \quad, \quad$ i.e., $m_{i, 2}\left(m_{N(\beta(i)-1), 1}\right)=v\left(\omega_{i}, \omega_{N(\beta(i)-1)}\right)$. Suppose that player $i$ is a buyer. Then, among these functions, only $g_{i}^{B, 2}$ and $x_{i}^{B, 2}$ depend on player $i^{\prime} s$ second bid $m_{i, 2}\left(m_{N(\beta(i)-1), 1}\right)$ and only on this bid. The specifications of $g_{i}^{B, 2}$ and $x_{i}^{B, 2}$, along with the truthful revelation via $m_{N(\beta(i)-1), 1}$, imply that player $i$ will choose $m_{i, 2}\left(m_{N(\beta(i)-1), 1}\right)$ to maximize $m_{i, 2}\left(m_{N(\beta(i)-1), 1}\right) v\left(\omega_{i}, \omega_{N(\beta(i)-1)}\right)-\frac{m_{N(\beta(i)-1), 1}^{2}}{2}$, i.e., $m_{i, 2}\left(m_{N(\beta(i)-1), 1}\right)=v\left(\omega_{i}, \omega_{N(\beta(i)-1)}\right)$. Hence, for every $i \in N$, if $s_{i}$ is twice iteratively undominated, then

$$
\begin{aligned}
& s_{i, 2}\left(\omega_{i}\right)\left(m_{N(\beta(i)-1), 1}\right)=v\left(\omega_{i}, \omega_{N(\beta(i)-1)}\right) \text { for all } \omega_{i} \in \Omega_{i} \\
& \text { if } m_{j, 1}=v\left(\omega_{j}\right) \text { for all } j \in N(\beta(i)-1) .
\end{aligned}
$$

Suppose that player $i$ is a seller. Then, among the functions $g_{i}^{D, l}$ and $x_{i}^{D, l}$ for all $(D, l) \in\{S, B\} \times\{1,2\}, g_{\beta(i)}^{3}$, and $x_{\beta(i)}^{3}$, only $g_{i}^{S, 3}, g^{3}, x_{i}^{S, 3}$, and $x_{i}^{3}$ depend on player i's third bid $m_{i, 3}\left(m_{N \backslash N(\beta(i)), 1}\right)$. Moreover, $g_{i}^{S, 3}$ and $x_{i}^{S, 3}$ depend only on this bid. The specifications of $g_{i}^{S, 3}$ and $x_{i}^{S, 3}$, along with the truthful revelation via $m_{N \backslash N(\beta(i)), 1}$, imply 
that the expected utility for player $i$ induced by $g_{i}^{S, 3}$ and $x_{i}^{S, 3}$ is

$$
m_{i, 3}\left(m_{N \backslash N(\beta(i)), 1}\right) v\left(\omega_{i}, \omega_{N \backslash N(\beta(i))}\right)+\frac{1-m_{N \backslash N(\beta(i)), 1}^{2}}{2},
$$

which is concave with respect to $m_{i, 3}\left(m_{N \backslash N(\beta(i)), 1}\right)$ and is maximized by

$$
m_{i, 3}\left(m_{N \backslash N(\beta(i)), 1}\right)=v\left(\omega_{i}, \omega_{N \backslash N(\beta(i))}\right) .
$$

Suppose that player $i$ is a buyer. Then, among these functions, only $g_{i}^{B, 3}, g^{3}, x_{i}^{B, 3}$, and $x_{i}^{3}$ depend on $m_{i, 3}\left(m_{N \backslash N(\beta(i)), 1}\right)$. Moreover, $g_{i}^{B, 3}$ and $x_{i}^{B, 3}$ depend only on $m_{i, 3}\left(m_{N \backslash N(\beta(i)), 1}\right)$. According to the specifications of $g_{i}^{B, 3}$ and $x_{i}^{B, 3}$, along with the truthful revelation via the first bids $m_{N \backslash N(\beta(i)), 1}$ by all the other groups, it follows that the expected utility for player $i$ induced by $g_{i}^{B, 3}$ and $x_{i}^{B, 3}$ is

$$
m_{i, 3}\left(m_{N \backslash N(\beta(i)), 1}\right) v\left(\omega_{i}, \omega_{N \backslash N(\beta(i))}\right)-\frac{m_{N \backslash N(\beta(i)), 1}^{2}}{2},
$$

which is concave with respect to $m_{i, 3}\left(m_{N \backslash N(\beta(i)), 1}\right)$ and is maximized by

$$
m_{i, 3}\left(m_{N \backslash N(\beta(i)), 1}\right)=v\left(\omega_{i}, \omega_{N \backslash N(\beta(i))}\right) .
$$

Fix $i \in N$ arbitrarily, and consider the expected utility for player $i$ induced by $g^{3}$ and $x_{i}^{3}$. Let $p=p(\beta(i)-1, m)$. From the specifications of $g^{3}$ and $x_{i}^{3}$, for each $\omega \in \Omega$, there exist $\bar{v}$ and $\tilde{v}$ such that

$$
\begin{aligned}
& g^{3}(m) v_{i}(\omega)-x_{i}^{3}(m)=\bar{v} \text { for all } m_{i, 3}\left(m_{N \backslash N(\beta(i)-1), 1}\right)>p, \\
& g^{3}(m) v_{i}(\omega)-x_{i}^{3}(m)=\tilde{v} \text { for all } m_{i, 3}\left(m_{N \backslash N(\beta(i)-1), 1}\right)<p, \\
& \min [\bar{v}, \tilde{v}] \leq g^{3}(m) v_{i}(\omega)-x_{i}^{3}(m) \leq \max [\bar{v}, \tilde{v}] \text { for } m_{i, 3}\left(m_{N \backslash N(\beta(i)-1), 1}\right)=p,
\end{aligned}
$$




$$
\bar{v}>\tilde{v} \quad \text { if } \quad v_{i}(\omega)>p,
$$

and

$$
\bar{v}<\tilde{v} \quad \text { if } \quad v_{i}(\omega)<p .
$$

From the above observations, it is clear that if all players play undominated strategies, then

$$
\begin{array}{r}
\min _{\omega_{N(\beta i) \backslash(i)}} v_{i}\left(\omega_{i}, \omega_{N \backslash\{i\}}\right) \leq m_{i, 3}\left(m_{N \backslash N(\beta(i)), 1}\right) \leq \max _{\omega_{N(\beta(i)) \backslash i\}}} v_{i}\left(\omega_{i}, \omega_{N \backslash\{i\}}\right) \\
\text { whenever } m_{j, 1}=v\left(\omega_{j}\right) \text { for all } j \in N \backslash N(\beta(i)) .
\end{array}
$$

Hence, we have proved that if all players play any twice iteratively undominated strategy profile $s \in S$, then, for every $i \in N$,

$$
\begin{aligned}
& s_{i, 1}\left(\omega_{i}\right)=v\left(\omega_{i}\right) \text { for all } \omega_{i} \in \Omega_{i}, \\
& s_{i, 2}\left(\omega_{i}\right)\left(m_{N(\beta(i)-1), 1}\right)=v\left(\omega_{i}, \omega_{N(\beta(i)-1)}\right) \text { for all } \omega_{i} \in \Omega_{i} \\
& \text { if } m_{j, 1}=v\left(\omega_{j}\right) \text { for all } j \in N(\beta(i)-1),
\end{aligned}
$$

and

$$
\begin{gathered}
\min _{\omega_{N(\beta(i)) \backslash i\}}} v_{i}\left(\omega_{i}, \omega_{N \backslash\{i\}}\right) \leq m_{i, 3}\left(m_{N \backslash N(\beta(i)), 1}\right) \leq \max _{\omega_{N(\beta(i))\{i\}}} v_{i}\left(\omega_{i}, \omega_{N \backslash\{i\}}\right) \\
\text { if } m_{j, 1}=v\left(\omega_{j}\right) \text { for all } j \in N \backslash N(\beta(i)) .
\end{gathered}
$$

\subsection{Competitive Allocations}

Since the size $2 n$ of each group is sufficiently large, it is almost certain from the law of large numbers and Assumptions 1 and 3 that $m_{i, 2}\left(m_{N(\beta(i)-1), 1}\right)=v\left(\omega_{i}, \omega_{N(\beta(i)-1)}\right)$ 
approximates $v_{i}\left(\omega_{0}, \omega_{i}\right)$, and from Assumption 2 that $p(\beta, m)$ approximates $p\left(\omega_{0}, e\right)$. Since the number $r$ of distinct groups is sufficiently large, it is almost certain that both $\min _{\omega_{N(\beta(i))\{i\}}} v_{i}\left(\omega_{i}, \omega_{N \backslash\{i\}}\right)$ and $\max _{\omega_{N(\beta(i))\{i\}}} v_{i}\left(\omega_{i}, \omega_{N \backslash\{i\}}\right)$ approximate $v_{i}\left(\omega_{0}, \omega_{i}\right)$, and therefore, $m_{i, 3}\left(m_{N \backslash N(\beta(i)), 1}\right)$ approximates $v_{i}\left(\omega_{0}, \omega_{i}\right)$. From the specifications of $\left(G^{(l)}\right)_{l=1}^{\infty}$, for sufficiently large $l$, the price-taking rule $\left(g^{3}, x^{3}\right)$ almost certainly determines the allocation, which implies that the probability of each player $i$ receiving one unit is close to 1 (close to zero) if $v_{i}\left(\omega_{0}, \omega_{i}\right)>p^{*}\left(\omega_{0}, e\right)$ (if $\left.v_{i}\left(\omega_{0}, \omega_{i}\right)<p^{*}\left(\omega_{0}, e\right)\right)$. The monetary transfer to seller $i$ approximates $p^{*}\left(\omega_{0} . e\right)$ (zero) if $v_{i}\left(\omega_{0}, \omega_{i}\right)<p^{*}\left(\omega_{0}, e\right)$ $\left(v_{i}\left(\omega_{0}, \omega_{i}\right)>p^{*}\left(\omega_{0}, e\right)\right)$. Moreover, the monetary transfer to buyer $i$ approximates $-p^{*}\left(\omega_{0}, e\right)$ (zero) if $v_{i}\left(\omega_{0}, \omega_{i}\right)>p^{*}\left(\omega_{0}, e\right) \quad\left(v_{i}\left(\omega_{0}, \omega_{i}\right)<p^{*}\left(\omega_{0}, e\right)\right)$. Thus, we have presented the full proof.

\section{References}

Abreu, D. and H. Matsushima (1992): "Virtual Implementation in Iteratively Undominated Strategies: Incomplete Information,” mimeo.

Abreu, D. and H. Matsushima (1994): "Exact Implementation,” Journal of Economic Theory 64, 1-19.

Bergemann, D. and S. Morris (2005a): "Robust Mechanism Design,” Econometrica 73, $1771-1813$. 
Bergemann, D. and S. Morris (2005b): "Robust Implementation: The Role of Large Type Spaces,” mimeo.

Camerer, C. (2003): Behavioral Game Theory, Russell Sage Foundation.

Fudenberg, D., M. Mobius, and A. Szeidl (2007): “Existence of Equilibrium in Large Double Auctions,” Journal of Economic Theory 133, 550-567.

Jackson, M. (1991): “Bayesian Implementation,” Econometrica 59, 461-477.

Klemperer, P. (2004): Auctions: Theory and Practice, Princeton University Press.

Krishna, V. (2002): Auction Theory, Academic Press.

Mas-Colell, A., M. Whinston, and J. Green (1995): Microeconomic Theory, Oxford University Press.

Maskin, E. and T. Sjöström (2002): “Implementation Theory,” in Handbook of Social Choice and Welfare Volume 1, ed. by K. Arrow, A. Sen, and K. Suzumura. Elsevier.

Matsushima, H. (1993): “Bayesian Monotonicity with Side Payments," Journal of Economic Theory 45, 128-144.

Matsushima, H. (2006a): “Role of Honesty in Full Implementation,” mimeo.

Matsushima, H. (2006b): “Mechanism Design with Side Payments: Individual Rationality and Iterative Dominance," forthcoming in Journal of Economic Theory.

McLean, R., J. Peck, and A. Postlewaite (2005): “On Price-Taking Behavior in Asymmetric Information Economics,” in Essays in Dynamic General Equilibrium: Festschrift for David Cass, ed. by A. Citanna, J. Donaldson, M. Polemarchakis, and S. Spear. Springer.

Milgrom, P. (2004): Putting Auction Theory to Work, Cambridge University Press. 
Palfrey, T. (1992): “Implementation in Bayesian Equilibrium: The Multiple Equilibrium Problem in Mechanism Design,” in Advances in Economic Theory: Sixth World Congress, ed. by J. J. Laffont. Cambridge University Press.

Palfrey, T. and S. Srivastava (1986): “Private Information in Large Economies,” Journal of Economic Theory 39, 34-58.

Reny, P. and M. Perry (2006): “Toward a Strategic Foundation for Rational Expectation Equilibrium,” Econometrica 74, 1231-1269. 